\title{
Hybrid Opportunistic Scheduling in Cognitive Radio Networks
}

\author{
Yang Li, Student Member, IEEE, and Aria Nosratinia, Fellow, IEEE
}

\begin{abstract}
In a cognitive (secondary) multiple-access network which is subject to interference power constraints imposed by a primary system, it is desirable to mitigate the interference on the primary and to harvest multiuser diversity gains in the secondary. To simultaneously achieve these goals, a twostep (hybrid) scheduling method is proposed that pre-selects a set of secondary users based on their interference on the primary, and from among them selects the user(s) that yield the highest secondary throughput. The optimal number of active secondary transmitters is characterized as a function of the primary interference constraint, the secondary transmit power, and the number of secondary transmitters $n$. The secondary sumrate (throughput) of the proposed algorithm grows optimally (proportional to $\log n$ ). We investigate the tradeoff between scaling the secondary throughput and reducing interference on the primary, and characterize the optimum tradeoff in the regime of large $n$. Finally, we study user scheduling under fairness constraints, which is necessary when the channel statistics of secondary nodes are not identical. A modified hybrid scheduling rule is proposed to ensure user fairness, while still achieving the optimal growth rate for the secondary throughput.
\end{abstract}

Index Terms-Spectrum sharing, opportunistic scheduling, cognitive radio, fairness.

\section{INTRODUCTION}

$\mathbf{U}$ NDER-UTILIZATION of spectrum [1] has motivated the study of various techniques that allow a set of secondary users to access the spectrum along with the primary users. Among different cognitive techniques [2], the underlay technique (also known as spectrum sharing) has attracted significant attention [3], [4]. Underlay cognitive radio allows the secondary users to share the spectrum if the interference caused on the primary is less than an interference temperature (threshold).

This paper studies an underlay cognitive multiple-access (MAC) channel with $n$ transmitters, in the presence of a primary system with $M_{p}$ transmitters and $N_{p}$ receivers. The primary and secondary systems are subject to mutual interference, where the secondary must comply with a set of interference power constraints imposed by the primary. The objective is to design a user scheduling method that exploits multiuser diversity in both cross links and secondary links, so that the secondary sum-rate (throughput) is maximized, while the interference induced on the primary is strictly bounded.

A brief overview of the past work is as follows. Zhang et al. [5] studied the power allocation of a secondary system

Manuscript received April 18, 2011; revised July 26, 2011 and September 30, 2011; accepted October 3, 2011. The associate editor coordinating the review of this paper and approving it for publication was M. Morelli.

The authors are with the University of Texas at Dallas, Richardson, TX 75080, USA (e-mail: \{yang, aria $\}$ utdallas.edu).

Digital Object Identifier 10.1109/TWC.2011.110811.110722 under various power and interference constraints. Multiple antennas at the secondary transmitter were exploited by [6] to balance the secondary throughput and the interference on the primary. Recently, ideas from opportunistic communication [7] have been applied in underlay cognitive radios. Tajer et al. [8] analyzed a parallel cognitive network and found a growth rate of $\Theta(\log \log n)$ for the throughput. The throughput limits of cognitive broadcast and MAC channel were analyzed by Li and Nosratinia [9], [10], where [9] randomly activates multiple secondary transmitters with interference smaller than a threshold. Jamal et al. [11] and Shen et al. [12] found that the secondary throughput can be increased by simultaneously activating as many secondary transmitters as possible. The multiuser diversity gain in cognitive networks was also studied by Hong et al. [13], Zhang et al. [14] and Ban et al. [15], showing that by selecting the secondary user with the highest signal-to-interference-and-noise ratio (SINR) under the primary interference constraints, the secondary throughput can grow as $\Theta(\log \log n)$.

The main results of this paper are as follows.

- We propose a two-step (hybrid) opportunistic scheduling that pre-selects a set of secondary transmitters with small interference, and from among them activates multiple transmitters with large secondary-channel gain. The preselection step provides cross-link diversity to minimize interference, while the second step provides multi-user diversity to improve the secondary throughput. The result is a throughput growing as $\Theta(\log n)$, which improves on the growth rate of $\Theta(\log \log n)$ in [13], [14], [15]. Furthermore, a $20-30 \%$ throughput gain is obtained compared with [9] for up to 200 secondary users. The proposed scheduling method is shown to be optimal asymptotically, and can reduce the interference on the primary proportionally to $n^{-q}$, while the secondary throughput grows proportionally to $\frac{1-q N_{p}}{N_{p}+1} \log n$, for $0 \leq q \leq \frac{1}{N_{p}}$.

- We characterize the (asymptotically) optimal number of active secondary transmitters as a function of the primary interference constraint, the secondary transmit power and $n$. To achieve the asymptotically optimal secondary throughput, the number of active transmitters must be proportional to $n^{\frac{1}{N_{p}+1}}$.

- The issue of fairness is studied; this issue arises when the node channel statistics are not identical. A method is proposed to ensure user fairness and the effect of a fairness constraint on asymptotic throughput is analyzed. It is shown that the modified scheduling method achieves the same optimal growth rate for the throughput, i.e., the fairness constraint does not affect the growth rate of the 


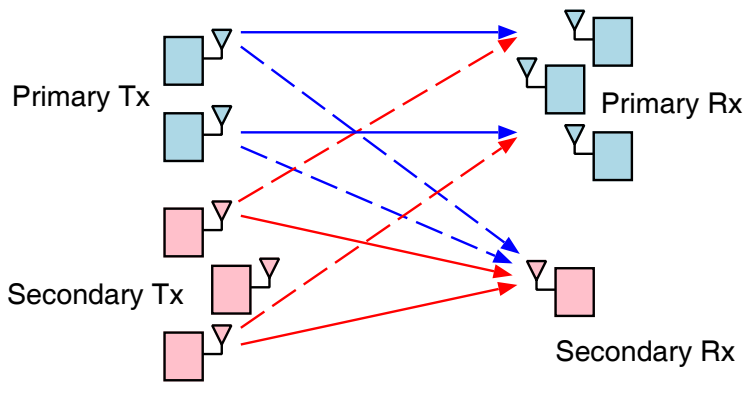

Fig. 1. Multiple access cognitive radio.

throughput for this algorithm.

The following asymptotic notations are used in this paper. For sufficiently large $n$,

$$
\begin{aligned}
& f(n)=O(g(n)): \quad \exists c_{1} \quad|f(n)|<c_{1}|g(n)| \\
& f(n)=\Theta(g(n)): \quad \exists c_{1}, c_{2} \quad c_{2}|g(n)|<|f(n)|<c_{1}|g(n)| \\
& f(n)=o(g(n)): \quad \forall \epsilon>0 \quad|f(n)|<\epsilon|g(n)|
\end{aligned}
$$

\section{System Model}

We consider a multiple-access (MAC) secondary system that coexists with a primary system, as shown in Figure 1. The primary system consists of $M_{p}$ transmitters and $N_{p}$ receivers, ${ }^{1}$ where each transmitter communicates with one or more receivers, and vice versa. The primary and secondary are subject to mutual interference from each other which is treated as noise. The interference from the secondary to each primary receiver must be smaller than a pre-defined interference temperature (threshold). For simplicity, all nodes are assumed to be single-antenna.

A block-fading channel model is assumed where all channel coefficients are independent, identically distributed (i.i.d.) circularly-symmetric complex Gaussian with zero mean and unit variance, denoted by $\mathcal{C} \mathcal{N}(0,1)$. For each transmission, a subset of secondary transmitters are activated; the collection of selected (active) transmitters is denoted by $\mathcal{S}$. The signal at the secondary receiver is:

$$
y=\sum_{i \in \mathcal{S}} \sqrt{P_{i}} h_{i} x_{i}+\sum_{\ell=1}^{M_{p}} \sqrt{P_{p}} g_{s, \ell} x_{p, \ell}+w,
$$

where $h_{i}$ is the channel coefficient from the secondary transmitter $i$ to the secondary receiver. The secondary transmitter $i$ sends a signal $x_{i}$ with power $P_{i}$, which is subject to a short term power constraint, i.e., $P_{i} \leq P$ for $1 \leq i \leq n$. The cross-channel coefficient from the primary transmitter $\ell$ to the secondary receiver is $g_{s, \ell}$. The primary transmitter $\ell$ sends a signal $x_{p, \ell}$ with power $P_{p}$ for $1 \leq \ell \leq M_{p}$. The additive noise $w$ has the distribution $\mathcal{C N}(0,1)$.

The interference power (caused by the secondary transmitters) on the primary receiver $j$ is

$$
I_{j}=\sum_{i \in \mathcal{S}} P_{i}\left|g_{j i}\right|^{2}
$$

\footnotetext{
${ }^{1}$ In this paper, $M_{p}$ and $N_{p}$ are assumed to be bounded, i.e., not scaling with $n$.
}

where $g_{j i}$ is the cross-channel coefficient from the secondary transmitter $i$ to the primary receiver $j$. For clarity of exposition, all the primary receivers are assumed to tolerate a shortterm interference power $\Gamma$; the case of unequal tolerances can be studied similarly (see Remark 1). We have

$$
I_{j} \leq \Gamma, \quad \text { for } \quad 1 \leq j \leq N_{p} .
$$

Throughout this paper, we assume the secondary receiver knows the secondary-channel coefficients $\left\{h_{i}\right\}$ but does not know any other channels (see Remark 2 for more details). We refer to the secondary forward channel simply as the secondary-channel, and the secondary cross-channel to the primary receiver as the cross-channel.

\section{Scheduling In Cognitive MAC Channel}

A scheduling scheme determines a set of active secondary transmitters $\mathcal{S}$ and their power $\left\{P_{i}\right\}_{i \in \mathcal{S}}$. The corresponding average secondary sum-rate (throughput) is given by

$$
R_{m a c}=\mathbb{E}\left[\log \left(1+\frac{G_{\text {sum }}}{1+I_{p}}\right)\right]
$$

where

$$
G_{\text {sum }}=\sum_{i \in \mathcal{S}} P_{i}\left|h_{i}\right|^{2}, \quad I_{p}=P_{p} \sum_{\ell=1}^{M_{p}}\left|g_{s, \ell}\right|^{2} .
$$

The statistics of $G_{\text {sum }}$ depends on the associated scheduling rule, and are independent of $I_{p}$, the interference from the primary.

\section{A. Hybrid Opportunistic Scheduling}

A secondary user scheduling should maximize the (average) secondary throughput, while satisfying the primary-imposed interference constraints. However, such two objectives often conflict. To increase the throughput, we want to activate many transmitters with large secondary-channel gains, but these transmissions may violate the interference constraints. Since the interference from various concurrent transmissions will add up, the scheduling of secondary transmitters is interdependent. We may choose many transmitters operating at low power, or a few transmitters at high power. Moreover, even for a fixed number of transmitters, reducing power from one transmitter allows increasing power from other transmitters. In general, the search for the optimal transmitter set and transmit power is a variation of the knapsack problem, which is NPcomplete. To simplify the problem, we adopt a decoupling power policy that is shown to be asymptotically optimal later on. This is an on-off power policy where each transmitter either operates at maximum power $P$ or remains silent. Then, the scheduling scheme is as follows:

1) Selection of Eligible Transmitters: The scheduling process has two parts. In its first part, we concentrate on limiting the interference, thus favoring transmitters with small crosschannel gains. Specifically, we only allow transmitters that do not violate an interference quota $\alpha$ on each primary receiver. The collection of such transmitters is defined as the eligible transmitter set:

$$
\mathcal{A}=\left\{i: P\left|g_{j i}\right|^{2}<\alpha, \forall 1 \leq j \leq N_{p}\right\} .
$$


This step can be considered as opportunistic interference avoidance. Recall that each primary receiver can tolerate interference power $\Gamma$. Once the maximum interference generated by each secondary transmitter is capped, the total interference at each primary receiver is guaranteed to be tolerable if no more than $k_{s}=\frac{\Gamma}{\alpha}$ eligible secondary transmitters are in operation. ${ }^{2}$

2) Selection of Active Transmitters: Now we choose from among the eligible transmitters those who will actually transmit. Up to $k_{s}$ secondary transmitters will be chosen that have high secondary-channel gains (SNRs), therefore producing multiuser diversity. The ordered channel gains of eligible transmitters are denoted by:

$$
\left|\tilde{h}_{1}\right|^{2} \geq\left|\tilde{h}_{2}\right|^{2} \geq \cdots \geq\left|\tilde{h}_{M}\right|^{2},
$$

where $\left|\tilde{h}_{i}\right|^{2}$ is the $i$ th largest channel gain of transmitters in $\mathcal{A}$, and $M=|\mathcal{A}|$ is the size of $\mathcal{A}$. Note that $M$ is a random variable. If $M>k_{s}$, the first $k_{s}$ transmitters in the above order will be active simultaneously. If $M \leq k_{s}$, then all the $M$ eligible transmitters will operate.

The above two-step scheme is called Hybrid Opportunistic Scheduling in the sense that it is driven by a hybrid of two criteria: Minimizing interference and maximizing throughput. This selection process requires neither exhaustive search nor joint power control among secondary transmitters, but it still guarantees compliance with the pre-defined interference threshold and captures the multiuser diversity gain. In addition, this scheduling is simple to design; the only parameter to consider is the interference quota $\alpha$ (thus $k_{s}$ ), which will be studied in the sequel.

Remark 1: Hybrid Opportunistic Scheduling still applies when primary receivers tolerate unequal amounts of interference, e.g., $\Gamma_{j}$ for $1 \leq j \leq N_{p}$. In this case, we design a separate interference quota for each primary receiver, i.e., $\alpha_{j}=\frac{\Gamma_{j}}{k_{s}}$, and re-define the eligible transmitter set as

$$
\mathcal{A}_{\text {neq }}=\left\{i: P\left|g_{j i}\right|^{2}<\alpha_{j}, \forall 1 \leq j \leq N_{p}\right\},
$$

such that the transmission of any $k_{s}$ eligible secondary transmitters complies with all the interference constraints. Notice that the selection of active transmitters is unaffected. One can show that most of the analysis and results in this paper still follow in a similar manner.

Remark 2: We briefly discuss the CSI requirement of the proposed scheme. First, each secondary transmitter compares its cross-channel gains ${ }^{3}$ to a threshold to evaluate its eligibility. Then, eligible transmitters each send 1-bit to inform the secondary receiver. The secondary-channel of eligible transmitters can be directly estimated at the receiver side. Therefore, this scheduling method requires little exchange of CSI. The thresholding operation of our method is essentially a

\footnotetext{
${ }^{2}$ For the purposes of analysis $\alpha$ is allowed to take any small and positive value, but for practical purposes it can be limited to the values that make $k_{s}$ to be an integer.

${ }^{3}$ The primary receiver emits packets for, e.g., handshake or ACK/NACK, which can be overheard by the secondary transmitter and used for crosschannel gain estimation in a TDD system. Also, under the spectrum leasing model [16], the primary receivers can be expected to actively promote spectrum reuse by transmitting pilots that can be used for cross-channel gain estimation. The latter model applies to both TDD and FDD.
}

distributed decision making process that significantly reduces the CSI overhead compared with methods that choose the least interfering secondary [11], because ranking is by necessity a centralized process and requires all nodes to communicate their cross-link to the receiver.

\section{B. Throughput Analysis}

Now we study the throughput achieved by the proposed Hybrid Opportunistic Scheduling. We first derive the average secondary throughput, and then maximize the throughput over $\alpha$. Under the proposed scheduling, we have

$$
G_{\text {sum }}=P \sum_{i=1}^{\min \left(k_{s}, M\right)}\left|\tilde{h}_{i}\right|^{2},
$$

which involves a sum of order statistics whose properties are given by the following lemma.

Lemma 1: Let $a$ and $b$ be large positive integers with $b \geq a$, and $S_{b}^{a}(\rho)$ be the sum of the highest $a$ order statistics out of $b$ i.i.d. exponentials with mean $\rho$. For any $0<\epsilon<1$,

$$
\begin{gathered}
\mathbb{P}\left(\left|S_{b}^{a}(\rho)-\rho \mu_{b}^{a}\right|<\epsilon \rho \mu_{b}^{a}\right)>1-O\left(\frac{1}{(\log b)^{2}}\right), \\
\mathbb{E}\left[S_{b}^{a}(\rho)\right]=\rho \mu_{b}^{a},
\end{gathered}
$$

where $\mu_{b}^{a}=a \log \frac{b}{a}+a+O(1)$.

Proof: See Appendix A.

Remark 3: In Lemma 1, $\mu_{b}^{a}$ can be considered as the multiuser diversity gain achieved by selecting the best $a$ out of $b$ users in i.i.d. Rayleigh fading channels. For $a=1$, it reduces to the case where one transmitter with the highest channel gain is selected, and we have $\mu_{b}^{1} \approx \log b$, a well known result [7], [17]. For $a=b$ (no selection), $S_{b}^{a}(\rho)$ obeys $\operatorname{Gamma}(b, \rho)$ distribution, and $\rho \mu_{b}^{b} \approx \rho b$.

Based on Lemma 1 and recalling that $k_{s}=\frac{\Gamma}{\alpha}$, for sufficiently small $\alpha$ (large $k_{s}$ ), we have the following results.

Theorem 1: Consider a secondary MAC with $n$ transmitters, each with power $P$. This MAC coexists with a primary system with $N_{p}$ receivers and $M_{p}$ transmitters each with power $P_{p}$. If each primary receiver tolerates interference power $\Gamma$, then the average secondary throughput $R_{\text {mac }}$ satisfies

$$
\begin{aligned}
R_{m a c} \geq & \log \frac{\left(\log n-\left(N_{p}+1\right) \log k_{s}+N_{p} \log (\Gamma / P)+1\right)}{1+M_{p} P_{p}} \\
& +\log \frac{P k_{s}}{1+M_{p} P_{p}}+O\left(\frac{1}{\log n}\right), \\
R_{m a c} \leq & \log \frac{\left(\log n-\left(N_{p}+1\right) \log k_{s}+N_{p} \log (\Gamma / P)+1\right)}{1+M_{p} P_{p}} \\
& +\log \frac{P k_{s}}{1+M_{p} P_{p}}+C_{0}+O\left(\frac{1}{\log n}\right),
\end{aligned}
$$

for sufficiently large $n$ and $k_{s}$, where $C_{0}=\log (\mathbb{E}[1 /(1+$ $\left.\left.\left.I_{p}\right)\right] \mathbb{E}\left[1+I_{p}\right]\right)$.

Proof: See Appendix B.

Remark 4: The lower bound (10) has only a constant gap $C_{0}$ relative to the upper bound (11) for large $n$, therefore, for given $k_{s}, R_{m a c}$ scales as $\log \log n$, similar to the results in [13], [14], [15]. To achieve this secondary rate, multiuser decoding is required at the secondary receiver for $k_{s}>1$, 
which is unlike TDMA scheduling $\left(k_{s}=1\right)$ where singleuser detection is sufficient. Finally, we note that $C_{0}$ depends only on the statistics of $I_{p}$, the interference from the primary to the secondary (see (5)).

Now, we design the interference quota $\alpha$ (equivalently $k_{s}$ ) to maximize the secondary throughput. Unlike conventional MAC where $k_{s}=n$ maximizes the sum throughput, in spectrum-sharing networks $k_{s}$ (thus $\alpha$ ) must be carefully designed due to the additional primary interference constraints. If $\alpha$ is very small, the number of eligible transmitters is also small on average, which reduces the multiuser diversity gain achieved by selecting from among the eligible transmitters. If $\alpha$ is very large, $\frac{\Gamma}{\alpha}$ will be small and few transmitters can be activated, thus once again the overall throughput will suffer. Therefore, it is desirable to optimize $\alpha$ (thus $k_{s}$ ), as shown by the following lemma.

Lemma 2: For sufficiently large $n$ the optimal number of active secondary transmitters $k_{s}^{o p t}$ satisfies

$$
\left|\frac{k_{s}^{o p t}}{k_{s}^{*}}-1\right| \leq \sqrt{1-\xi}
$$

where $k_{s}^{*}=\left(\frac{\Gamma}{P e}\right)^{\frac{N_{p}}{N_{p}+1}} n^{\frac{1}{N_{p}+1}}$ and $\xi$ is given by (52).

Proof: See Appendix C.

Lemma 2 asymptotically bounds the optimal number of active secondary transmitters as a function of $\Gamma, P$ and $n$. It shows that, essentially, $k_{s}^{o p t}$ cannot be too far from $k_{s}^{*}$. Motivated by this lemma, we choose $k_{s}=k_{s}^{*}$ and in the following theorem obtain a throughput growth rate that is later shown to be asymptotically optimal (see Section III-C).

Theorem 2: Consider a secondary MAC with $n$ transmitters each with power $P$. This MAC coexists with a primary system with $N_{p}$ receivers and $M_{p}$ transmitters with power $P_{p}$. If each primary receiver tolerates interference power $\Gamma$, then the average secondary throughput $R_{m a c}$ satisfies

$$
\begin{aligned}
& R_{\text {mac }} \geq \frac{1}{N_{p}+1} \log n+C_{1}+O\left(\frac{1}{\log n}\right), \\
& R_{\text {mac }} \leq \frac{1}{N_{p}+1} \log n+C_{1}+C_{0}+O\left(\frac{1}{\log n}\right),
\end{aligned}
$$

for sufficiently large $n$ by activating $k_{s}^{*}$ transmitters, where $C_{1}=\frac{N_{p}}{N_{p}+1} \log \frac{\Gamma}{P e}+\log \frac{\left(N_{p}+1\right) P}{1+M_{p} P_{p}}$.

Proof: Notice that the proof of Theorem 1 holds for $k_{s}=\Theta\left(n^{1 /\left(N_{p}+1\right)}\right)$. The theorem follows by substituting $k_{s}^{*}$ into (10) and (11), respectively.

The implications of Theorem 2 are as follows. Intuitively, the secondary throughput is reduced when the number of primary receivers (constraints) increases. Theorem 2 explicitly quantifies this: $R_{m a c}=\frac{1}{N_{p}+1} \log n+O(1)$. For small $N_{p}$, Hybrid Opportunistic Scheduling achieves a (significant) fraction of the throughput of an ordinary MAC, if $n$ is large enough. The achieved throughput is proven to be optimal asymptotically (with $n$ ) in the sequel.

So far we have shown that the multiuser nature of a secondary system can improve the secondary throughput. In fact, this multiuser flexibility can also be used to mitigate the interference on the primary. A tradeoff exists between the primary interference reduction and the secondary throughput enhancement under Hybrid Opportunistic Scheduling, which is described as follows.
Corollary 1: Consider the allowable interference on each primary receiver being bounded as $\Theta\left(n^{-q}\right)$. Then, the average secondary throughput satisfies

$$
R_{\text {mac }}=\frac{1-q N_{p}}{N_{p}+1} \log n+O(1),
$$

for sufficiently large $n$ under Hybrid Opportunistic Scheduling, where $0 \leq q \leq \frac{1}{N_{p}}$.

Proof: Notice that Theorem 2 holds for $\Gamma=\Theta\left(n^{-q}\right)$. The Corollary follows by substituting $\Gamma$ into the lower and upper bounds given by (13).

Based on Corollary 1, as $n$ increases, Hybrid Opportunistic Scheduling can mitigate interference (to zero) on the primary receivers, while the secondary throughput grows as $\Theta(\log n)$. The allowable interference $\Gamma$ is made to decline as $\Theta\left(n^{-q}\right)$, which leads $R_{m a c}$ to decrease linearly in $q$. If $\Gamma$ is reduced more slowly, e.g., decreasing as $\Theta\left(\frac{1}{\log n}\right)$, the secondary throughput can increase at a rate of $\frac{1}{N_{p}+1} \log n$. If we try to mitigate the primary interference faster than $\Theta\left(n^{-q}\right)$, i.e., $q \geq \frac{1}{N_{p}}$, the secondary throughput only grows as $o(\log n)$. Therefore, as $N_{p}$ increases, not only the throughput of the secondary decreases, but also its ability of reducing the interference on the primary.

Remark 5: The key to the secondary growth rate $\Theta(\log n)$ is to activate multiple secondary transmitters while limiting the interference. This approach is in contrast with [13], [14], [15] where a single transmitter with the highest SNR was activated. The main questions to be answered in this work have been: how many secondary transmitters we should activate, how to choose the active secondary transmitters in a relatively straight forward fashion, and how much power should the active transmitters emit to achieve the growth rate while satisfying the interference constraint.

\section{Optimality of Hybrid Opportunistic Scheduling}

We first find an upper bound for the average secondary throughput that applies regardless of transmission strategies.

Theorem 3: Consider the coexistence of a secondary MAC with $n$ transmitters and a primary system with $N_{p}$ receivers. The maximum average throughput of the secondary, $R_{\text {mac }}^{o p t}$, satisfies

$$
R_{m a c}^{o p t} \leq \frac{1}{N_{p}+1} \log n+O(\log \log n) .
$$

\section{Proof: See Appendix D.}

The gap between the above upper bound and the throughput attained by Hybrid Opportunistic Scheduling (shown in Theorem 2) is only on the order of $O(\log \log n)$. This gap is negligible relative to $\Theta(\log n)$ for sufficiently large $n$, therefore, Hybrid Opportunistic Scheduling asymptotically attains the maximum throughput:

$$
\lim _{n \rightarrow \infty} \frac{R_{m a c}}{R_{m a c}^{o p t}}=1 .
$$

Remark 6: The growth rate $\Theta(\log n)$ can also be attained by activating secondary users simply according to the least interference, i.e., only based on cross-channel gains [9], [18]. The similarity of growth rates may tempt one to say that there 
is no gain in utilizing secondary channel information [18]. However, similarity of growth rates hides $o(\log n)$ throughput gains by the two-step (hybrid) approach that are highly nontrivial and practically important. For instance, our results show throughput gains of around 20-30\% over [9] (see Figure 3) by selecting the users with large secondary-channel gain.

\section{Scheduling Under Non-I.I.D. Link Statistics}

In this section, we consider a network where neither the secondary-channels nor cross-channels are identically distributed. This is a practical scenario due to, e.g., different path losses for various links. Assuming that the channel gains obey one out of a finite number of distributions, we enumerate them with the variable $d \in\{1, \ldots, D\}$. Specifically, each user has a secondary-channel gain and cross-channel gain that obeys the exponential distribution with parameter $\rho_{d}$ and $\lambda_{d}$, respectively. The number of users in each of these groups is $\beta_{d} n$, where $\sum_{d=1}^{D} \beta_{d}=1$.

The secondary transmitters that enjoy larger $\rho_{d}$ and smaller $\lambda_{d}$ have a higher probability to be active under Hybrid Opportunistic Scheduling, so user fairness is no longer guaranteed. In the following, we extend Hybrid Opportunistic Scheduling to ensure a (long-term) temporal fairness [19], [20] in the sense that each secondary transmitter has equal probability (time fraction) to be active. For clarity of exposition, we consider $M_{p}=N_{p}=1$, i.e., one pair of primary transmitter and receiver.

Our strategy is to design the interference quota for Group $d$ to be proportional to $\lambda_{d}$, such that all transmitters have equal eligible probability. More precisely, the interference quota for Group $d$ is

$$
\alpha_{d}=\frac{\lambda_{d} \Gamma}{k_{s} \sum_{j=1}^{D} \beta_{j} \lambda_{j}}, \quad \text { for } 1 \leq d \leq D .
$$

The corresponding eligible transmitter set for Group $d$ is

$$
\mathcal{A}_{d}=\left\{i: P\left|g_{j i}\right|^{2}<\alpha_{d}, \forall 1 \leq j \leq N_{p}, i \in \text { Group } d\right\} .
$$

Therefore, the eligible probability of any transmitter is

$$
p^{\prime} \approx \frac{\Gamma}{k_{s} P \sum_{d=1}^{D} \beta_{j} \lambda_{j}} .
$$

Then, we separately select (up to) $\beta_{d} k_{s}$ eligible transmitters from among each group. One can verify that the above modifications ensure both the fairness requirement and the primary interference restriction. We have the following lemma:

Lemma 3: For the network described above, the average secondary throughput $R_{m a c}$ satisfies

$$
\begin{aligned}
R_{m a c} \geq & \log \frac{P k_{s}\left(\log \frac{n p^{\prime}}{k_{s}}+1\right)}{1+P_{p}}+\log \sum_{d=1}^{D} \rho_{d} \beta_{d}+O\left(\frac{1}{\log n}\right) \\
R_{m a c} \leq & \log \frac{P k_{s}\left(\log \frac{n p^{\prime}}{k_{s}}+1\right)}{1+P_{p}}+\log \sum_{d=1}^{D} \rho_{d} \beta_{d} \\
& +C_{0}+O\left(\frac{1}{\log n}\right)
\end{aligned}
$$

for sufficiently large $n$ under the modified Hybrid Opportunistic Scheduling.
Proof: For brevity we only provide an outline. First, note that the user selection is decoupled among different groups. Let $M_{d}$ be the number of eligible transmitters for Group $d$, then $M_{d}$ is binomially distributed with parameter $\left(\beta_{d} n, p^{\prime}\right)$ (similar to (27)). In this case, $G_{\text {sum }}$ is a mixture of sums of order statistics described by Lemma 1, i.e., $G_{\text {sum }}=$ $\sum_{d=1}^{D} S_{M_{d}}^{\beta_{d} k_{s}}\left(\rho_{d}\right)$ in distribution given $M_{d}$ sufficiently large. The rest of the proof is similar to Theorem 1 .

With slight modification of Lemma 2, we choose the number of active secondary transmitters as:

$$
k_{s}^{*}=\underbrace{\sqrt{\frac{\Gamma}{e P \sum_{d=1}^{D} \beta_{d} \lambda_{d}}}}_{c^{\prime}}(n)^{\frac{1}{2}} .
$$

The above equation indicates that as the average cross-channel gain $\sum_{d} \beta_{d} \lambda_{d}$ increases, fewer secondary transmitters should be activated simultaneously. Notice that $k_{s}^{*}$ becomes identical to that given by Lemma 2 (with $N_{p}=1$ ) when $\lambda_{d}=1$ for $1 \leq d \leq D$. Based this choice of $k_{s}$ and Lemma 3, we obtain the following results.

Theorem 4: For the network described above, the average secondary throughput $R_{\text {mac }}$ satisfies

$$
\begin{aligned}
& R_{\text {mac }} \geq \frac{1}{2} \log n+C_{2}+O\left(\frac{1}{\log n}\right), \\
& R_{\text {mac }} \leq \frac{1}{2} \log n+C_{2}+C_{0}+O\left(\frac{1}{\log n}\right),
\end{aligned}
$$

for sufficiently large $n$ by activating $k_{s}=c^{\prime} \sqrt{n}$ transmitters, where $C_{2}=\log \frac{\sum_{d} \beta_{d} \rho_{d}}{\sqrt{\sum_{d} \beta_{d} \lambda_{d}}}+\log \frac{2}{1+P_{p}} \sqrt{\frac{\Gamma P}{e}}$.

Proof: The theorem follows by substituting $k_{s}=c^{\prime} \sqrt{n}$ into Lemma 3.

Remark 7: From Theorem 4, the growth rate of $R_{\text {mac }}$ is $\frac{1}{2} \log n$, which is optimal and thus is unaffected due to the imposition of the fairness constraint. Besides the growth rate, the impact of channel heterogeneity on the secondary throughput can also be seen by inspecting $C_{2}$ : The lower (upper) bound of the throughput increases with the average secondary-channel gain, $\sum_{d} \beta_{d} \rho_{d}$, but decreases with the $a v$ erage cross-channel gain, $\sum_{d} \beta_{d} \lambda_{d}$. Intuitively, as $\sum_{d} \beta_{d} \lambda_{d}$ increases, statistically, the secondary transmitters more easily cause interference on the primary, thus fewer of them can be active simultaneously, which in turn leads to a smaller secondary throughput. Finally, note that Theorem 4 includes, as a special case, the results of Theorem 2 when the primary system simply consists of one transmitter-receiver pair.

\section{Numerical Results}

In this section, we illustrate our results with simulations. We use $P_{p}=P=10$. Unless otherwise specified, $M_{p}=N_{p}=1$ and the allowable interference power on the primary receiver is $\Gamma=5$. All simulations are averaged over $2 \times 10^{4}$ channel realizations.

Figure 2 shows the (asymptotically) optimal number of active secondary transmitters characterized by Lemma 2 . The throughput achieved by activating $k_{s}^{*}=\left\lceil\sqrt{\frac{\Gamma}{P e} n}\right\rceil$ transmitters surpasses (or equals) that achieved by activating fixed $k_{s}$ transmitters, for $n$ from 20 to around 400. Although Lemma 2 only 


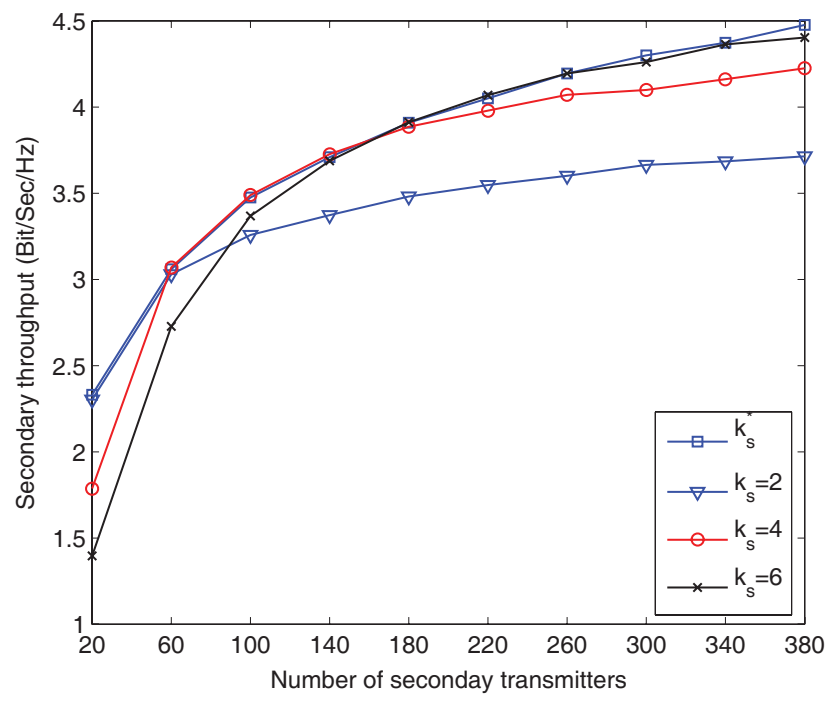

Fig. 2. Optimal number of active secondary transmitters.

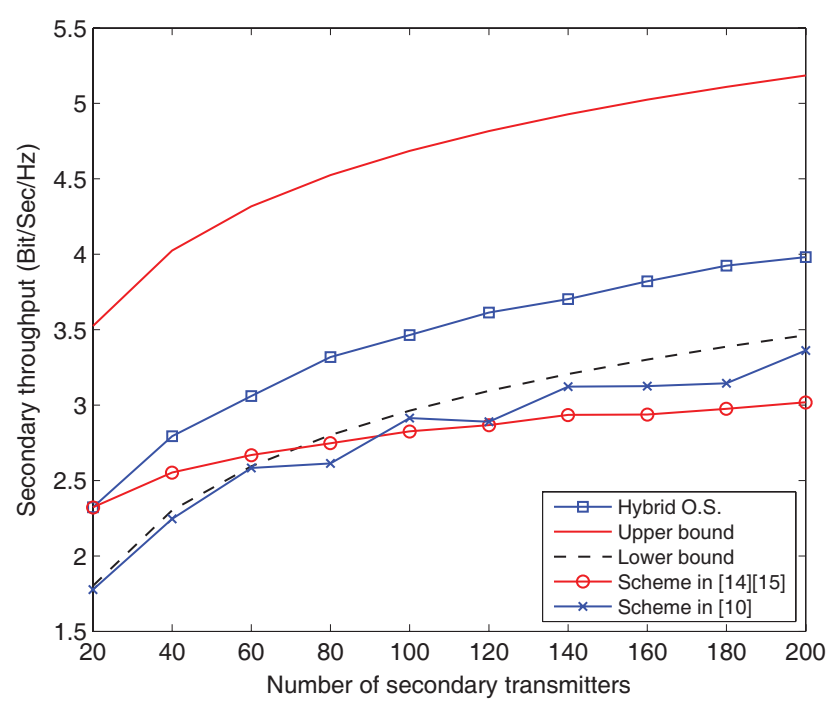

Fig. 3. Throughput of Hybrid Opportunistic Scheduling and other schemes.

suggests $k_{s}^{\text {opt }}$ cannot be far away from $k_{s}^{*}$, simulations imply that $k_{s}^{*}$ may be indeed optimal. Intuitively, as $n$ increases, the number of secondary transmitters that have desirably small cross-channel gains also increases on average, therefore, more secondary transmitters should be active simultaneously.

Figure 3 illustrates Theorem 2 and compares Hybrid Opportunistic Scheduling with several other schemes. The throughput of the proposed method is bounded by the asymptotic bounds in Theorem 2, even for small $n$. Hybrid Opportunistic Scheduling attains a throughput higher than that attained in [9], where the transmitters are selected only based on cross-channels without considering the secondary-channel conditions. Also, the achieved throughput is higher than that achieved in [14], [15] where the (single) secondary transmitter with the highest SINR is activated. The throughput of the proposed method scales as $\Theta(\log n)$, which is faster than the $\Theta(\log \log n)$ growth achieved in [14], [15]. Figure 4 shows the impact on the secondary throughput of the primary network $\left(M_{p}\right.$ and $\left.N_{p}\right)$. As $M_{p}$ increases, due to experiencing

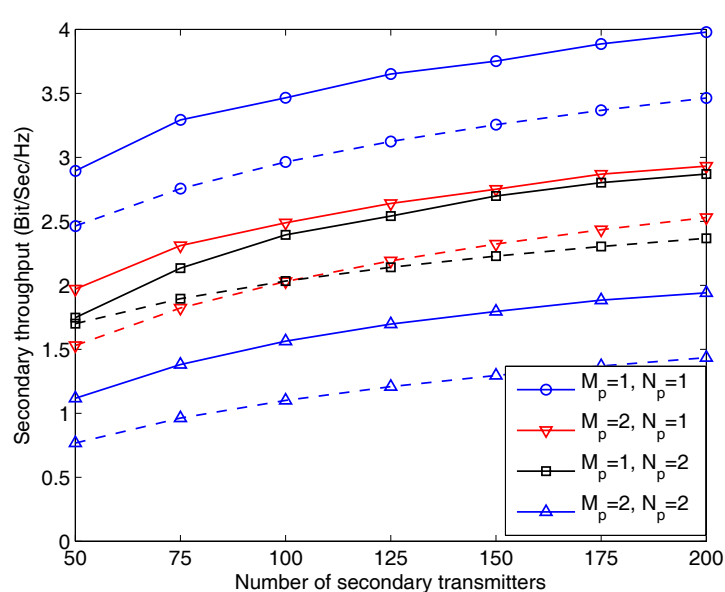

Fig. 4. Throughput of Hybrid Opportunistic Scheduling under different $M_{p}$ and $N_{p}$. The dash lines correspond to the asymptotic lower bound derived by Theorem 2 .
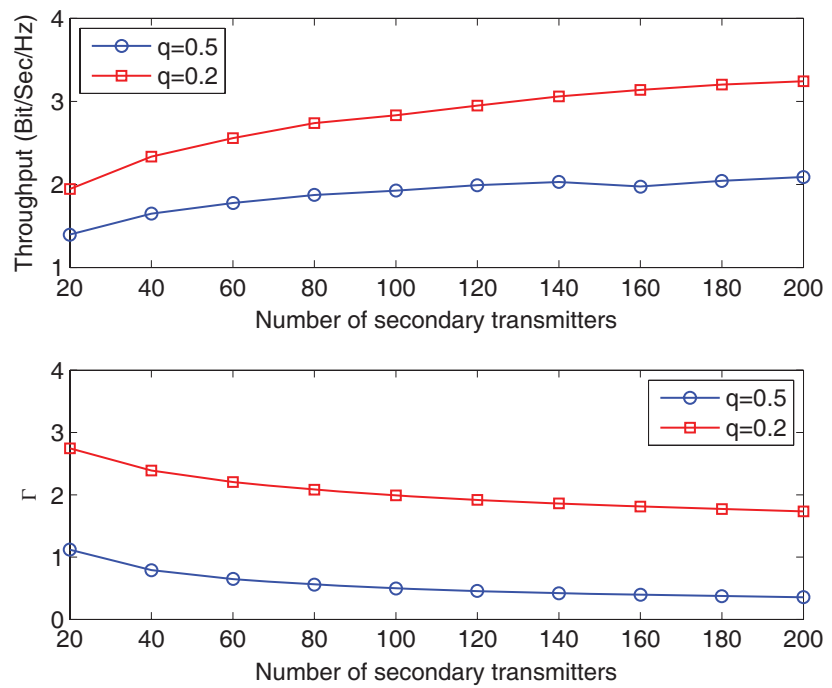

Fig. 5. Throughput versus transmitter number, vanishing $\Gamma$.

more interference from the primary, the secondary throughput decreases. As $N_{p}$ increases, due to more constraints imposed by the primary, the secondary throughput again decreases.

The results of Corollary 1 are illustrated by Figure 5. The allowable interference power $\Gamma$ declines (to zero) as $n^{-q}$, while the throughput still grows logarithmically with $n$. In addition, one can see the tradeoff given by Corollary 1: For larger $q$, the interference power decreases faster but the secondary throughput increases more slowly, and vice versa.

Figure 6 and Figure 7 show the performance of Hybrid Opportunistic Scheduling under non-i.i.d. links. Here, $n=50$ and $k_{s}=4 ; D=2$ and $\beta_{1}=\beta_{2}=0.5$, i.e., two groups with equal number of transmitters. Figure 6 shows the secondary throughput for the case of $\rho_{1}=\lambda_{1}=\lambda_{2}=1$ and $\rho_{2}=2$ (non-i.i.d. secondary-channels), and for the case of $\rho_{1}=\rho_{2}=$ $\lambda_{1}=1$ and $\lambda_{2}=2$ (non-i.i.d. cross channels). With the fairness constraint, the modified Hybrid Opportunistic Scheduling still attains a throughput that is very close to that attained without any fairness restriction. Figure 7 shows the ratio of average portion of active time of Group 1 and Group 2. If this ratio 


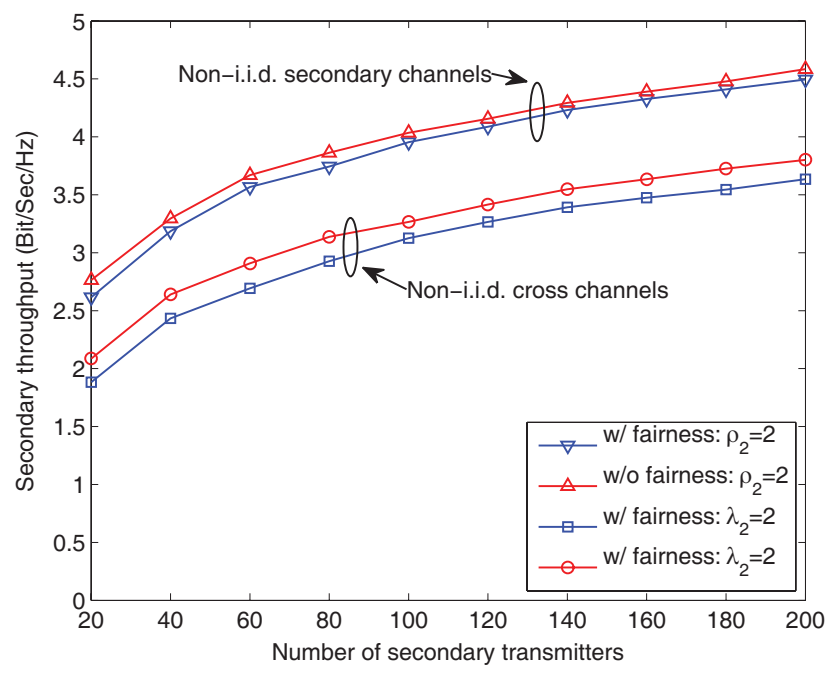

Fig. 6. Throughput of Hybrid Opportunistic Scheduling under a fairness constraint.
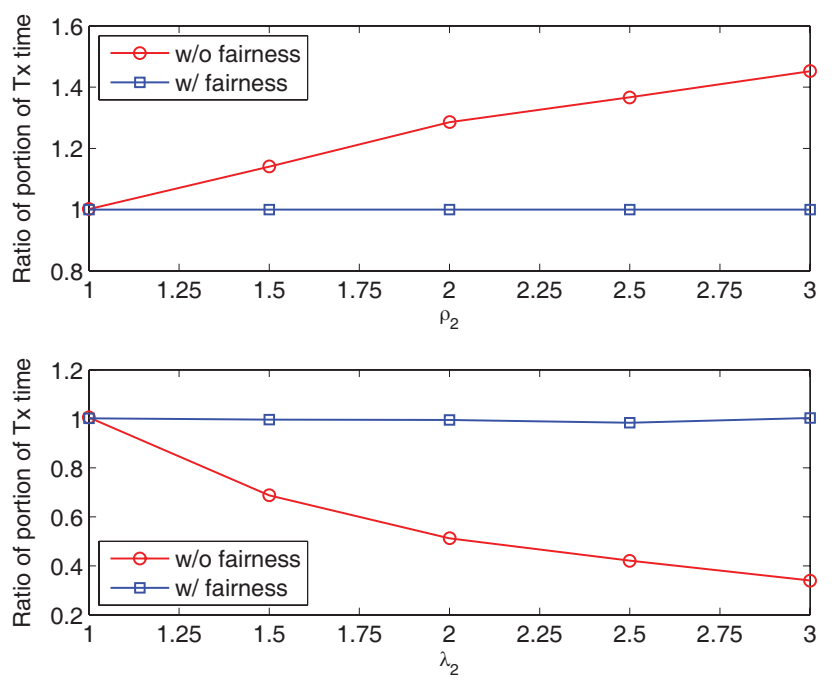

Fig. 7. Fairness metric for Hybrid Opportunistic Scheduling.

equals 1 , each transmitter has an equal portion of active time and the system is temporally fair [19]. The larger the ratio, the larger portion of active time of Group 2 relative to that of Group 1. One can see that user fairness is ensured under the modified scheduling.

\section{CONCLUSION}

In this paper, we investigate user scheduling in cognitive MAC networks and propose Hybrid Opportunistic Scheduling, which is driven by two objectives: Maximizing the secondary throughput and minimizing the primary interference. The proposed scheme strictly controls the primary interference by opportunistic interference avoidance, and enhances the secondary throughput by activating transmitters with large secondary-channel gain. We characterize the optimal number of active secondary transmitters and a tradeoff between the secondary throughput enhancement and the primary interference reduction. Finally, we study user scheduling under a fairness constraint when links have non-i.i.d. statistics.

\section{APPENDIX A}

PROOF OF LEMMA 1

Proof: Let $Z_{1}, \cdots, Z_{b}$ be i.i.d. exponentials with mean $\rho$. From [17], we know that $S_{b}^{a}(\rho)$ has the same distribution as

$$
\sum_{i=1}^{b-a} \frac{a}{b-i+1} Z_{i}+Z_{b-a+1}+\cdots+Z_{b} .
$$

Therefore we can calculate its expectation:

$$
\mathbb{E}\left[S_{b}^{a}(\rho)\right] \triangleq \rho \mu_{b}^{a}=\rho a\left(\sum_{i=1}^{b} \frac{1}{i}-\sum_{i=1}^{a} \frac{1}{i}\right)+\rho a .
$$

It is known [21] that, for any positive integer $k$,

$$
\log k+\gamma+\frac{1}{2(k+1)}<\sum_{i=1}^{k} \frac{1}{i}<\log k+\gamma+\frac{1}{2 k}
$$

where $\gamma$ is the Euler constant. Hence, for sufficiently large $b$ and $a(b \geq a)$, we obtain

$$
\mu_{b}^{a}=a \log \frac{b}{a}+a+O(1) .
$$

Now, we calculate the variance of $S_{b}^{a}(\rho)$. From (22), we have:

$$
\operatorname{Var}\left[S_{b}^{a}(\rho)\right]<(\rho a)^{2} \sum_{i=a+1}^{b} \frac{1}{(i-1) i}+\rho^{2} a<2 \rho^{2} a .
$$

Applying the Chebyshev inequality, for any $0<\epsilon<1$, we have

$$
\mathbb{P}\left(\left|S_{b}^{a}(\rho)-\rho \mu_{b}^{a}\right|>\epsilon \rho \mu_{b}^{a}\right)<\frac{\operatorname{Var}\left[S_{b}^{a}(\rho)\right]}{\left(\epsilon \rho \mu_{b}^{a}\right)^{2}}<O\left(\frac{1}{(\log b)^{2}}\right) .
$$

The above second inequality holds for any $a=O\left(b^{\delta}\right)$ and $\delta<1$. The lemma follows by taking the complement of the random event in inequality.

\section{APPENDIX B \\ PROOF OF THEOREM 1}

Proof: To begin with, note that $M$ (the size of $\mathcal{A}$ ) is binomially distributed with parameter

$$
p=\left(1-e^{-\frac{\alpha}{P}}\right)^{N_{p}},
$$

since $\left\{\left|g_{j i}\right|^{2}\right\}$ are i.i.d. exponentials with unit mean. For any $0<\epsilon_{1}<1$, we have

$$
\mathbb{P}\left(|M-n p|>\epsilon_{1} n p\right)<\frac{(1-p)}{\epsilon_{1}^{2} p n}=O\left(\frac{1}{n}\right)
$$

based on the Chebyshev inequality. For convenience, we denote

$$
n_{1}=\left\lfloor\left(1-\epsilon_{1}\right) n p\right\rfloor, \quad n_{2}=\left\lceil\left(1+\epsilon_{1}\right) n p\right\rceil .
$$

Then, from (28), we have

$$
\mathbb{P}\left(M \geq n_{1}\right)>1-O\left(\frac{1}{n}\right), \quad \mathbb{P}\left(M \geq n_{2}\right)<O\left(\frac{1}{n}\right) .
$$

Now, we establish a lower bound. Based on (4), $R_{m a c}$ depends two independent random variables $I_{p}$ and $G_{\text {sum }}$, where $I_{p}$ is distributed as $\operatorname{Gamma}\left(M_{p}, P_{p}\right)$, and given $M=m$, $G_{\text {sum }}$ has the same distribution as $S_{m}^{k_{s}}(P)$ for $m \geq k_{s}$ (see 
Lemma 1). Condition on $I_{p}=x$ and expand the conditional throughput $R_{\text {mac } \mid I_{p}}(x)$ :

$$
\begin{aligned}
R_{\text {mac } \mid I_{p}}(x) & =\sum_{m=1}^{n} \mathbb{E}\left[\log \left(1+\frac{G_{\text {sum }}}{1+x}\right) \mid M=m\right] \mathbb{P}(M=m) \\
& \geq \sum_{m=n_{1}}^{n} \mathbb{E}\left[\log \left(1+\frac{S_{m}^{k_{s}}(P)}{1+x}\right)\right] \mathbb{P}(M=m),
\end{aligned}
$$

where the inequality holds since we discard non-negative terms associated with $m<n_{1}$ in the summation and $n_{1}>k_{s}$ for sufficiently large $n$. For any $0<\epsilon<1$, we further expand (31) by conditioning on the event $\mathcal{C}_{m}=\left\{S_{m}^{k_{s}}(P)>\right.$ $\left.(1-\epsilon) P \mu_{m}^{k_{s}}\right\}$ :

$$
\begin{aligned}
& R_{\text {mac } \mid I_{p}}(x) \\
& \geq \sum_{m=n_{1}}^{n} \mathbb{E}\left[\log \left(1+\frac{S_{m}^{k_{s}}(P)}{1+x}\right) \mid \mathcal{C}_{m}\right] \mathbb{P}(M=m) \mathbb{P}\left(\mathcal{C}_{m}\right) \\
& >\sum_{m=n_{1}}^{n} \log \left(1+\frac{(1-\epsilon) P \mu_{m}^{k_{s}}}{1+x}\right) \mathbb{P}(M=m) \\
& \quad \times\left(1-O\left(\frac{1}{(\log n)^{2}}\right)\right) \\
& >\log \left(1+\frac{(1-\epsilon) P \mu_{n_{1}}^{k_{s}}}{1+x}\right) \mathbb{P}\left(M \geq n_{1}\right)\left(1-O\left(\frac{1}{(\log n)^{2}}\right)\right) \\
& >\log \left(1+\frac{(1-\epsilon) P \mu_{n_{1}}^{k_{s}}}{1+x}\right)\left(1-O\left(\frac{1}{n}\right)\right)\left(1-O\left(\frac{1}{(\log n)^{2}}\right)\right) .
\end{aligned}
$$

To obtain (32), we use the result from Lemma 1 by noting $m=\Theta(n)$ for $m \geq n_{1}$ :

$$
\mathbb{P}\left(\mathcal{C}_{m}\right) \geq 1-O\left(\frac{1}{(\log n)^{2}}\right) .
$$

We have (33), since $\mu_{n_{1}}^{k_{s}} \leq \mu_{m}^{k_{s}}, \forall m \geq n_{1}$. Finally, (34) uses (30).

From Lemma 1 and the fact that $n_{1}=\Theta(n)$, we have $\mu_{n_{1}}^{k_{s}}=O(\log n)$. Since $\log (1+z)=\log z+\log \left(1+\frac{1}{z}\right)$ for $z>0$, we expand the right hand side of (34):

$$
R_{m a c \mid I_{p}}(x)>\log \frac{P(1-\epsilon) \mu_{n_{1}}^{k_{s}}}{1+x}+O\left(\frac{1}{\log n}\right) .
$$

Take expectation with respect to $I_{p}$ and use the convexity of $h(z)=\log \left(1+\frac{c_{1}}{c_{2}+z}\right)$ :

$$
\begin{aligned}
\mathbb{E}\left[R_{\text {mac } \mid I_{p}}(x)\right] & >\log \frac{P(1-\epsilon) \mu_{n_{1}}^{k_{s}}}{1+\mathbb{E}\left[I_{p}\right]}+O\left(\frac{1}{\log n}\right) \\
& =\log \frac{P \mu_{n_{1}}^{k_{s}}}{1+P_{p} M_{p}}+\log (1-\epsilon)+O\left(\frac{1}{\log n}\right) .
\end{aligned}
$$

Finally, we calculate $\mu_{n_{1}}^{k_{s}}$. Since $\alpha=\frac{\Gamma}{k_{s}}$, from (27), we have $p \approx\left(\frac{\Gamma}{k_{s} P}\right)^{N_{p}}$ for large $k_{s}$. From Lemma 1, we have

$$
\mu_{n_{1}}^{k_{s}}=k_{s}\left(\log \frac{n \Gamma^{N_{p}}}{P^{N_{p}} k_{s}^{N_{p}+1}}+1\right)+O(1) .
$$

Substituting (38) into (37), and with some calculation, we have the desired lower bound in (10).
Now, we find an upper bound. Let $T=\frac{1}{1+I_{p}}$ and $R_{m a c \mid T}(t)$ be the conditional throughput. Expand $R_{\operatorname{mac} \mid T}(t)$ based on the event $\left\{M \leq n_{2}\right\}$ and its complement:

$$
\begin{aligned}
R_{\text {mac } \mid T}(t)= & \mathbb{E}\left[\log \left(1+t G_{\text {sum }}\right) \mid M \leq n_{2}\right] \mathbb{P}\left(M \leq n_{2}\right) \\
& +\mathbb{E}\left[\log \left(1+t G_{\text {sum }}\right) \mid M>n_{2}\right] \mathbb{P}\left(M>n_{2}\right) \\
\leq & \log \left(1+t \mathbb{E}\left[G_{\text {sum }} \mid M \leq n_{2}\right]\right) \\
& +\log \left(1+t \mathbb{E}\left[G_{\text {sum }} \mid M>n_{2}\right]\right) \mathbb{P}\left(M>n_{2}\right)
\end{aligned}
$$

where (39) uses the Jensen inequality. Since $\mathbb{E}\left[G_{\text {sum }} \mid M=i\right]$ is a non-decreasing function of $i$, we have

$$
\begin{aligned}
R_{\text {mac } \mid T}(t) \leq & \log \left(1+t \mathbb{E}\left[S_{n_{2}}^{k_{s}}(P)\right]\right) \\
& +\log \left(1+t \mathbb{E}\left[S_{n}^{k_{s}}(P)\right]\right) \mathbb{P}\left(M>n_{2}\right) \\
< & \log \left(1+t P \mu_{n_{2}}^{k_{s}}\right)+\log \left(1+t P \mu_{n}^{k_{s}}\right) O\left(\frac{1}{n}\right),
\end{aligned}
$$

where (40) uses (30). Take expectation with respect to $T$.

$$
\begin{aligned}
\mathbb{E}\left[R_{\text {mac|T }}(t)\right] \leq & \log \left(1+P \mu_{n_{2}}^{k_{s}} \mathbb{E}[T]\right) \\
& +\log \left(1+P \mu_{n}^{k_{s}} \mathbb{E}[T]\right) O\left(\frac{1}{n}\right) \\
< & \log \left(1+P \mu_{n_{2}}^{k_{s}} \mu_{T}\right)+O\left(\frac{\log \log n}{n}\right) \\
= & \log P \mu_{n_{2}}^{k_{s}}+\log \mu_{T} \\
& +\log \left(1+O\left(1 / \mu_{n_{2}}^{k_{s}}\right)\right)+O\left(\frac{\log \log n}{n}\right),
\end{aligned}
$$

where $\mu_{T}=\mathbb{E}[T]$. The Jensen inequality is used in (41) and the identity $\log (1+z)=\log z+\log \left(1+\frac{1}{z}\right)$ for $z>0$ is used in (43). Similar to (38), we have

$$
\mu_{n_{2}}^{k_{s}}=k_{s}\left(\log \frac{n \Gamma^{N_{p}}}{P^{N_{p}} k_{s}^{N_{p}+1}}+1\right)+O(1) .
$$

Substituting (44) into (43), we obtain the desired upper bound in (11) with $C_{0}=\log \left(\mu_{T}\left(1+M_{p} P_{p}\right)\right)$. Notice that $C_{0} \geq 0$, because

$$
\mu_{T}\left(1+M_{p} P_{p}\right)=\mathbb{E}\left[1 /\left(1+I_{p}\right)\right] \mathbb{E}\left[1+I_{p}\right] \geq 1,
$$

where the equality holds if and only if $I_{p}$ is a constant.

\section{APPENDIX C}

PROOF OF LEMMA 2

Proof: The exact expression of $R_{m a c}$ as a function of $k_{s}$ is unknown in Theorem 1, thus a direct maximization of $R_{\text {mac }}\left(k_{s}\right)$ is impossible. The idea of this proof is to (approximately) optimize bounds on $R_{m a c}$ and show that the resulting answer is sufficient for our purposes. We begin with the lower and upper bounds in Theorem 1, denoted as $L\left(k_{s}\right)$ and $U\left(k_{s}\right)$, which can be written as (ignoring vanishing terms):

$$
\begin{aligned}
& L\left(k_{s}\right)=\log r\left(k_{s}\right)+\log \frac{P}{1+M_{p} P_{p}}, \\
& U\left(k_{s}\right)=\log r\left(k_{s}\right)+\log \frac{P}{1+M_{p} P_{p}}+C_{0},
\end{aligned}
$$


where

$$
r\left(k_{s}\right)=k_{s}\left(-\left(N_{p}+1\right) \log k_{s}+\log n(\Gamma / P)^{N_{p}}+1\right) .
$$

Notice that $L\left(k_{s}\right)$ and $U\left(k_{s}\right)$ are identical function of $k_{s}$ except a constant gap $C_{0}$. Intuitively, the value of $k_{s}$ that maximizes $L\left(k_{s}\right)$ (or $U\left(k_{s}\right)$ ), denoted by $k_{s}^{*}$, should also (almost) maximize $R_{\text {mac }}\left(k_{s}\right)$. We justify this intuition in the rest of the proof.

First, we find $k_{s}^{*}$. Since $\log (\cdot)$ is a monotonic-increasing function, we maximize $r\left(k_{s}\right)$ instead. For the asymptotic analysis, $k_{s}$ can be considered as a continuous variable. So, solving $r^{\prime}(\cdot)=0$, we obtain:

$$
k_{s}^{*}=\left(\frac{\Gamma}{P e}\right)^{\frac{N_{p}}{N_{p}+1}} n^{\frac{1}{N_{p}+1}} .
$$

Now, consider $k_{s}=k_{s}^{1}$ such that $\log r\left(k_{s}^{1}\right)+C_{0}<$ $\log r\left(k_{s}^{*}\right)$. Then, $k_{s}^{1}$ is not the maximizer of $R_{\text {mac }}\left(k_{s}\right)$, because in this case $U\left(k_{s}^{1}\right)<L\left(k_{s}^{*}\right)$, which implies $R_{m a c}\left(k_{s}^{1}\right)<$ $R_{\text {mac }}\left(k_{s}^{*}\right)$. Therefore, $k_{s}^{o p t}$, the true maximizer of $R_{m a c}\left(k_{s}\right)$, must satisfy

$$
\log r\left(k_{s}^{o p t}\right)+C_{0} \geq \log r\left(k_{s}^{*}\right) .
$$

Let $k_{s}^{o p t}=k_{s}^{*}+\Delta k$. From (50), with some algebra, we have:

$$
|\Delta k| \leq \sqrt{1-\xi} k_{s}^{*}
$$

where

$$
\xi=\exp \left(-C_{0}\right)=\left(\mu_{T}\left(1+P_{p} M_{p}\right)\right)^{-1} .
$$

Numerically, one can see that $\xi \approx 1$ and thus $\Delta k \approx 0$. For example, if $P_{p}=10, \xi \approx 0.8$ for $M_{p}=4$ and $\xi \approx 0.9$ for $M_{p}=8$.

\section{APPENDIX D \\ PROOF OF THEOREM 3}

Proof: Consider an arbitrary $\mathcal{S}$ and $\left\{P_{i}\right\}_{i \in \mathcal{S}}$ that comply with the interference constraints imposed by the primary. We first enlarge the secondary throughput by assuming zero interference from the primary:

$$
\begin{aligned}
R_{\text {mac }} & \leq \log \left(1+\sum_{i \in \mathcal{S}} P_{i}\left|h_{i}\right|^{2}\right) \\
& \leq \log \left(1+G_{\max } P_{\text {sum }}\right),
\end{aligned}
$$

where

$$
P_{\text {sum }}=\sum_{i \in \mathcal{S}} P_{i}, \quad G_{\max }=\max _{1 \leq i \leq n}\left|h_{i}\right|^{2} .
$$

Now we find an upper bound for $R_{\text {mac }}$ regardless of transmission strategies. First, we bound $P_{\text {sum }}$ and formulate an optimization problem:

$$
\begin{gathered}
\max _{\mathcal{S},\left\{P_{i}\right\}} P_{\text {sum }} \\
\text { s.t. }: \sum_{i \in \mathcal{S}} P_{i}\left|g_{j i}\right|^{2} \leq \Gamma \text { for } 1 \leq j \leq N_{p}, \text { and } P_{i} \leq P .
\end{gathered}
$$

which is a standard linear programming whose solution is denoted by $P_{\text {sum }}^{*}$. Here, $P_{\text {sum }}^{*}$ is a random variable depending on the channel realizations. A direct solution requires joint optimization over $\mathcal{S}$ and $\left\{P_{i}\right\}$, but a simpler analysis exists for upper bounds. We relax the set of interference constraints in (56) to a single sum constraint, which never decreases $P_{\text {sum }}^{*}$ :

$$
\sum_{i \in \mathcal{S}} P_{i} I_{\text {sum }, i} \leq N_{p} \Gamma
$$

where

$$
I_{\text {sum }, i}=\sum_{j=1}^{N_{p}}\left|g_{j i}\right|^{2}
$$

is the total cross-channel gains from the secondary transmitter $i$ to all the primary receivers. Thus, $\left\{I_{\text {sum }, i}\right\}_{i=1}^{n}$ are i.i.d. $\operatorname{Gamma}\left(N_{p}, 1\right)$. We order $I_{s u m, i}$ among all the secondary transmitters:

$$
\tilde{I}_{\text {sum }, 1} \leq \cdots \leq \tilde{I}_{\text {sum }, n} .
$$

Then, we construct the following problem by further relaxing the constraint of (57):

$$
\begin{gathered}
\max _{\mathcal{S},\left\{P_{i}\right\}} P_{\text {sum }} \\
\text { s.t. : } \sum_{i=1}^{|\mathcal{S}|} P_{i} \tilde{I}_{\text {sum }, i} \leq N_{p} \Gamma \text { and } P_{i} \leq P .
\end{gathered}
$$

The solution for the above problem, denoted by $P_{s u m, 1}^{*}$, is always greater than or equal to $P_{\text {sum }}^{*}$. The corresponding $\left\{P_{i}\right\}$ achieves $P_{\text {sum }, 1}^{*}$ are in form of $P_{i} \geq P_{j}$ for $i \leq j$. Thus, we have

$$
P_{\text {sum }, 1}^{*} \leq P N_{\text {smax }}
$$

where $N_{\text {smax }}$ is the maximum possible value of $|\mathcal{S}|$ that satisfies

$$
P \sum_{i=1}^{|\mathcal{S}|-1} \tilde{I}_{\text {sum }, i} \leq N_{p} \Gamma .
$$

For brevity, we outline the rest of the proof. It can be shown that $N_{\text {smax }}$ converges to $\Theta\left(n^{\frac{1}{N_{p}+1}}\right)$ in probability. Because $P_{\text {sum }}^{*} \leq P N_{s}$ and $G_{\max }$ (the maxima of $n$ i.i.d. exponentials) scales as $\log n$ [17], [7], we have (see (54)):

$$
\begin{aligned}
R_{\text {mac }}^{\text {opt }} & \leq \log \left(\Theta\left(n^{\frac{1}{N_{p}+1}}\right) \log n\right) \\
& =\frac{1}{N_{p}+1} \log n+O(\log \log n) .
\end{aligned}
$$

\section{REFERENCES}

[1] Federal Communications Commission, "Facilitating opportunities for flexible, efficient, and reliable spectrum use employing cognitive radio technologies," Dec. 2003.

[2] S. A. Jafar, S. Srinivasa, I. Maric, and A. Goldsmith, "Breaking spectrum gridlock with cognitive radios: an information theoretic perspective," Proc. IEEE, vol. 97, no. 5, pp. 894-914, May 2009.

[3] M. Gastpar, "On capacity under receive and spatial spectrum-sharing constraints," IEEE Trans. Inf. Theory, vol. 53, no. 2, pp. 471-487, Feb. 2007.

[4] A. Ghasemi and E. S. Sousa, "Fundamental limits of spectrum-sharing in fading environments," IEEE Trans. Wireless Commun., vol. 6, no. 2, pp. 649-658, Feb. 2007

[5] R. Zhang, S. Cui, and Y.-C. Liang, "On ergodic sum capacity of fading cognitive multiple-access and broadcast channels," IEEE Trans. Inf. Theory, vol. 55, no. 11, pp. 5161-5178, Nov. 2009.

[6] R. Zhang and Y.-C. Liang, "Exploiting multi-antennas for opportunistic spectrum sharing in cognitive radio networks," IEEE J. Sel. Topics Signal Process., vol. 2, no. 1, pp. 88-102, Feb. 2008.

[7] P. Viswanath, D. N. C. Tse, and R. Laroia, "Opportunistic beamforming using dumb antennas," IEEE Trans. Inf. Theory, vol. 48, no. 6, pp. $1277-$ 1294, June 2002. 
[8] A. Tajer and X. Wang, "Multiuser diversity gain in cognitive networks with distributed spectrum access," in Proc. Information Sciences and Systems, Mar. 2009, pp. 135-140.

[9] Y. Li and A. Nosratinia, "Capacity limits of multiuser multiantenna cognitive networks," IEEE Trans. Inf. Theory, submitted, available: http://arxiv.org/pdf/1008.3641.

[10] — , "Opportunistic cognitive radio broadcast channel: asymptotic performance," Globecom Workshop on Mobile Computing and Emerging Communication Networks, Dec. 2010.

[11] N. Jamal, H. E. Saffar, and P. Mitran, "Throughput enhancements in point-to-multipoint cognitive systems," in Proc. ISIT, June 2009, pp. 2742-2746.

[12] C. Shen and M. Fitz, "Opportunistic spatial orthogonalization and its application in fading cognitive radio networks," IEEE J. Sel. Topics Signal Processing, to appear.

[13] J.-P. Hong and W. Choi, "Capacity scaling law by multiuser diversity in cognitive radio systems," in Proc. ISIT, June 2010, pp. 2088-2092.

[14] R. Zhang and Y.-C. Liang, "Investigation on multiuser diversity in spectrum sharing based cognitive radio networks," IEEE Commun. Lett., vol. 14, no. 2, pp. 133-135, Feb. 2010.

[15] T. W. Ban, W. Choi, B. C. Jung, and D. K. Sung, "Multi-user diversity in a spectrum sharing system," IEEE Trans. Wireless Commun., vol. 8, no. 1, pp. 102-106, Jan. 2009.

[16] S. Jayaweera and T. Li, "Dynamic spectrum leasing in cognitive radio networks via primary-secondary user power control games," IEEE Trans. Wireless Commun., vol. 8, no. 6, pp. 3300-3310, June 2009.

[17] H. A. David and H. N. Nagaraja, Order Statistics. Wiley, 2003.

[18] N. Jamal, H. E. Saffar, and P. Mitran, "Asymptotic scheduling gains in point-to-multipoint cognitive networks." Available: http://arxiv.org/pdf/ 1001.3365

[19] X. Liu, E. K. P. Chong, and N. B. Shroff, "A framework for opportunistic scheduling in wireless networks," Computer Networks, vol. 41, no. 4, pp. 451-474, 2003.

[20] S. Patil and G. De Veciana, "Measurement-based opportunistic scheduling for heterogenous wireless systems," IEEE Trans. Commun., vol. 57, no. 9, pp. 2745-2753, Sep. 2009.
[21] S. Sanayei and A. Nosratinia, "Opportunistic downlink transmission with limited feedback," IEEE Trans. Inf. Theory, vol. 53, no. 11, pp. 4363-4372, Nov. 2007.

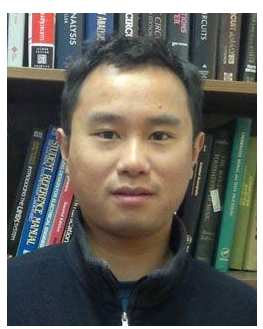

Yang Li (S'10) received his B.S. and M.S. degree in electrical engineering from Shanghai Jiao Tong University, Shanghai, China in 2005 and 2008, respectively. He is currently pursuing the Ph.D degree in electrical engineering at the University of Texas at Dallas. He was an intern at Huawei Technologies Co. Ltd during 2008 and 2011. His current interests include cognitive radio, heterogeneous networks, interference management and cooperative communication.

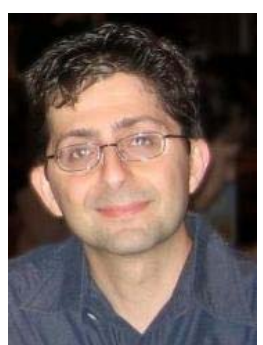

Aria Nosratinia (S'87,M'97,SM'04,F'10) is Jonsson Distinguished Professor of Electrical Engineering at the School of Engineering and Computer Science, the University of Texas at Dallas. He received his $\mathrm{Ph} . \mathrm{D}$. in Electrical and Computer Engineering from the University of Illinois at Urbana-Champaign in 1996. He has held visiting appointments at Princeton University, Rice University, and UCLA. His interests lie in the broad area of information theory and signal processing, with applications in wireless communications. He is associate editor for the IEEE TRansactions on Wireless Communications. He was an officer and member of the Board of Governors of the IEEE Information Theory Society in 2010-2011 and served as treasurer for ISIT 2010 in Austin. $\mathrm{He}$ has been an editor for the IEEE TRANSACTIONS ON INFORMATION Theory, IEEE Transactions on Image Processing, IEEE Signal Processing Letters, and IEEE Wireless Communications Magazine. He has been the recipient of the National Science Foundation career award, and is a fellow of IEEE. 